\title{
Acknowledgment to Reviewers of Journal of Intelligence in 2021
}

\author{
Journal of Intelligence Editorial Office
}

MDPI AG, St. Alban-Anlage 66, 4052 Basel, Switzerland

Rigorous peer-reviews are the basis of high-quality academic publishing. Thanks to the great efforts of our reviewers, Journal of Intelligence was able to maintain its standards for the high quality of its published papers. Thanks to the contribution of our reviewers, in 2021, the median time to first decision was 38 days. The editors would like to extend their gratitude and recognition to the following reviewers for their precious time and dedication, regardless of whether the papers they reviewed were finally published:

Adam B. Lockwood

Adam Chuderski

Alexander Christensen

Alissa Beath

Andre Beauducel

André Kretzschmar

Andrea Trubanova Wieckowski

Andreas Demetriou

Andrew Conway

Antonella Lopez

Athanasios Koutras

Boris Forthmann

Branton Shearer

Bruno Dauvier

Carmen Ferrandiz

Casey L. Brown

Catrinel Haught Tromp

Connie S. Barber

Dana Joseph

Daniel Simonet

David Aly Redvaldsen

David C. Geary

David Cropley

Diane Halpern

Elizabeth Austin

Emanuel Jauk

Ernesto San Martin

Estefanía Martín

Esther Keulers

Eunice Gaerlan-Price

Evgenia Gkintoni

Florian Schmitz

Francisco Villegas Lirola

George Spanoudis

Gizem Hülür
Gordon B. Schmidt

Ian Deary

Isaac Nana Akuffo

Jan-Philipp Freudenstein

Jeffrey M. Gredlein

Jens F. Beckmann

Jens Lange

Jillian Lauer

John Mayer

Jonas Lang

Jonathan Plucker

Jonathan Wai

Jose M. Mestre

Juan Wang

Judith A. Hall

Judith Glück

Julian Schulze

Justin Brienza

Kamila Urban

Karl Schweizer

Kees Jan Kan

Kimberly Wingert

Kristina Meyer

Kristof Kovacs

Larry M. Starr

Ludivine Martin

María José Gutiérrez Cobo

Marianne Nolte

Mark Leikin

Marta Bistron

Martha Michalkiewicz

Matthias Ziegler

Mercedes Ferrando

Michael Lewis

Michèle N. Schubiger 
Miguel-Ángel Esteban-Navarro

Mihaela Flavia Avram

Moshe Zeidner

Nicholas F. Benson

Oliver Wilhelm

Paola Aiello

Paul Silvia

Phillip L. Ackerman

Qin Zhao

Rachel Wilson

Rex Jung

Robert Sternberg

Rogier A. Kievit

Rory MacLean

Roza Leikin

Rui Abrunhosa Gonçalves

Sara Hart
Sarah Davis

Selcuk Acar

Serena Mastria

Sergio Agnoli

Sergio Morra

Silvia Postigo-Zegarra

Simonetta D'Amico

Stefano A. Cerri

Stephen Skalicky

Steven Umbrello

Thierry Lecerf

Tobias Debatin

Toni Schmader

Torberg Falch

Ulrich Schroeders

Vivian Ciaramitaro 